\title{
DNA-VLP prime-boost intra-nasal immunization induces cellular and humoral anti-HIV-1 systemic and mucosal immunity with cross-clade neutralizing activity
}

\author{
L Buonaguro, C Devito, ML Tornesello, U Schröder, B Wahren, \\ Jorma Hinkula and FM Buonaguro
}

\section{Linköping University Post Print}

N.B.: When citing this work, cite the original article.

Original Publication:

L Buonaguro, C Devito, ML Tornesello, U Schröder, B Wahren, Jorma Hinkula and FM Buonaguro, DNA-VLP prime-boost intra-nasal immunization induces cellular and humoral anti-HIV-1 systemic and mucosal immunity with cross-clade neutralizing activity, 2007, Vaccine, (25), 32, 5968-5977.

http://dx.doi.org/10.1016/j.vaccine.2007.05.052

Copyright: Elsevier http://www.elsevier.com/

Postprint available at: Linköping University Electronic Press http://urn.kb.se/resolve?urn=urn:nbn:se:liu:diva-38564 


\section{DNA-VLP prime-boost intra-nasal immunization induces cellular and humoral anti-HIV-1 systemic and mucosal immunity with cross-clade neutralizing activity.}

L. Buonaguro ${ }^{1}$, C. Devito ${ }^{2}$, M. Tagliamonte ${ }^{1}$, M.L. Tornesello ${ }^{1}$, U. Schröder $^{3}$, B. Wahren ${ }^{4,5}$, J. Hinkula ${ }^{2}$ and F.M. Buonaguro ${ }^{1 *}$.

${ }^{1}$ Viral Oncogenesis and Immunotherapies \& AIDS Reference Center, Ist. Naz. Tumori "Fond. G. Pascale", Naples - Italy; ${ }^{2}$ Department for Molecular Virology, IMK, Faculty of Health Science, Linköping University, Linköping - Sweden; ${ }^{3}$ Eurocine Vaccines AB, Karolinska Science Park, Stockholm - Sweden; ${ }^{4}$ Department of Microbiology, Tumor and Cell Biology, Karolinska Institute, Stockholm - Sweden; ${ }^{5}$ Swedish Institute for Infectious Disease Control, Stockholm - Sweden.

*Corresponding author:

F.M. Buonaguro, M.D.

Head

Viral Oncogenesis and Immunotherapies \& AIDS Reference Center

Ist. Naz. Tumori "Fond. G. Pascale"

Via Mariano Semmola, 1

80131 NAPLES - ITALY

Tel. +39-081-5903.830

Fax. +39-081-545.1276

E-mail: irccsvir@unina.it 


\begin{abstract}
The immune response to HIV-1 Virus-Like Particles (VLPs), presenting a clade A Ugandan gp120, has been evaluated in a mouse model by intranasal (i.n.) administration by a VLP+VLP homologous or a DNA+VLP heterologous prime-boost immunization protocol, including a HIV-1 DNA gp160/rev plasmid. Furthermore, the effect of the Eurocine lipidbased mucosal L3 adjuvant on the VLP immunogenicity has been assessed as well.

The designed heterologous protocol is able to increase the env-specific humoral and cellular immune response, compared to the homologous protocol, which is to some extent increased by the administration of L3-adjuvanted VLP boosting dose. The anti-gag response is statistically increased in both homologous and heterologous protocols, particularly when the VLP boosting dose is adjuvanted.

Immune sera from immunized animals exhibit $>50 \%$ ex vivo neutralizing activity against heterologous A and B-clade viral isolates. An envelope B-cell epitope mapping shows an enhanced response against V3 epitopes all across the C2-V5 region in the heterologous prime-boost immunization strategy.

The induction of humoral immunity at mucosal sites, which represents the main port of entry for the HIV-1 infection, is extremely relevant. In this framework, the DNA-VLP heterologous prime-boost protocol appears a promising preventive vaccine approach which can significantly benefit from specific mucosal adjuvants, as the Eurocine L3.
\end{abstract}

Keywords: HIV-1, Virus-Like Particles (VLP), prime-boost approach, mucosal adjuvants. Running title: DNA-VLP mucosal administration in adjuvants. 


\section{INTRODUCTION}

Virus-like particles (VLPs) represent an antigen presenting and delivery system currently under investigation as potential vaccine for different human viruses, such as Hepatitis, Papilloma, Rotavirus, Parvovirus and Norwalk virus [1-6]. The HIV-targeted VLPs (HIVVLPs) are based on the HIV-1 Pr55gag precursor protein property to assemble as immature, non-replicating and non-infectious VLPs with an effective induction of both arms of the immune response [7-13]. In particular, the enveloped HIV-VLPs developed in our laboratory present an entire gp120 molecule derived from an Ugandan HIV-1 isolate of the clade $A$, identified in our laboratory $[11 ; 14 ; 15]$. The HIV-VLP ${ }_{A}$ S show a strong in vivo immunogenicity in Balb/c mice, in absence of adjuvants, and HIV-1-specific CTLs as well as cross-clade neutralizing antibodies, active on primary HIV-1 isolates, have been detected in immunized animals [12]. Furthermore, the immunogenicity of the HIV-VLP ${ }_{A} S$ has been evaluated in Balb/c mice by intra-nasal (i.n.) routes, in a homologous (VLP+VLP) primeboost protocol without any adjuvant formulation, showing the induction of humoral IgA and/or IgG antibody response with neutralizing activity, in sera and at mucosal (vaginal and intestinal) sites, as well as cellular immune response [16].

Heterologous prime-boost vaccination strategies have been originally developed to improve the CD8+ T cell response, combining a DNA or VLP priming with live-vector boosters. This was based on the rationale that the DNA or VLPS are able to drive epitopes into the MHC class I pathway and to make the immune response focused only on the desired vaccine antigen, while the live-vector booster enhances this immune response by both expressing large amounts of antigen and stimulating a pro-inflammatory response $[17 ; 18]$. However, several observations indicate that, although cell-mediated immunity is crucial for controlling and eradicating viral infections, neutralizing antibodies are pivotal in reducing the infectivity titer of the initial HIV-1 inoculum (potentially to sterilization) as well as neutralizing free virions during the first rounds of replication. This greatly facilitates the outcome of the second line of defense operated by effector T cells that are required to 
eliminate virus-infected cells[19-22]. Consequently, anti-HIV-1 prime-boost vaccination strategies should include a protein component to induce an optimal titer of neutralizing antibodies $[23 ; 24]$. In this context, a VLP boosting would represent an extremely appropriate choice, given the several advantages over the single recombinant protein vaccines. In particular, 1 ) to present envelope antigens in their native form; 2) to enter into professional antigen-presenting cells (APCs) by either phagocytosis or receptormediated uptake; 3 ) to activate the endogenous as well as exogenous pathways leading to the presentation of viral antigens by both MHC class I and class II molecules $[13 ; 25]$. This results in induction of both arms of immune response and, specifically, effective neutralizing antibodies directed against conformational epitopes. A strategy based on DNA priming and VLP boosting has been previously shown to elicit a strong systemic neutralizing humoral response when administered intramuscularly [26] or intradermally [27].

Considering that the transmission of HIV-1 infection during heterosexual or homosexual intercourse accounts for as much as $80 \%$ of AIDS globally, a specific mucosal immunity is extremely relevant for controlling the primary HIV-1 transmission. This can be achieved by mucosally delivered vaccines which, besides the advantage of conferring mucosal as well as systemic immunity, show an increased stability and allow the elimination of needles. This, especially in developing countries, may significantly reduce the risk of further spreading HIV-1 infection. Moreover, the immunogenic efficacy of mucosal vaccines would greatly benefit from the co-administration with mucosal adjuvants that can initiate and support the transition from innate to adaptive immunity.

In this respect, the Eurocine $\mathrm{L3}$ is a potent mucosal adjuvant for protein vaccines [28], based on mono-olein/oleic acid vesicles approved for human use as a nasal vaccine adjuvant and already employed in a phase I clinical trial, performed in Sweden, for nasal immunization with diphtheria vaccine. Moreover, it has been evaluated in intra-nasal immunization protocol with heat-killed Mycobacterium bovis bacilli Calmette-Guerin (BCG), inducing levels of protection comparable to the conventional live BCG vaccine [29]. 
In the present study, the mucosal immunogenicity of HIV-VLPAs has been evaluated in a homologous (VLP+VLP) and heterologous (DNA+VLP) prime-boost strategy by intra-nasal administration. In order to achieve a broader neutralizing activity, a multi-clade approach has been designed employing envelope molecules of B (DNA) and A (VLP) subtypes. Furthermore, the enhancing effect of the L3 adjuvant on the HIV-VLP $\mathrm{A}_{\mathrm{A}}$ immunogenicity has been assessed as well. 


\section{MATERIALS AND METHODS}

\subsection{Immunization experiments.}

Female Balb/c mice, each group consisting of six animals 6-8 weeks of age, were intranasally immunized with $8 \mu \mathrm{g}$ HIV-1 gp160/rev DNA mixed with 2\% N3 adjuvant [30] or with $2 \mu \mathrm{g}$ rVLPgag/gp160 clade A mixed with PBS or 2\% L3-adjuvant. After 4 weeks, mice were intranasally boostered with 2 ug rVLPgag/gp120 clade A mixed with PBS or $2 \%$ L3-adjuvant. For both primary and booster immunizations, mice were anastezised for 2 min with Isofurane gas and immunogen (6-7 $\mu \mathrm{l} /$ nose nare) was given in the nose at a total volume of $14 \mu \mathrm{l} /$ mouse. The mice were awake approx. 1 min after receiving the immunogen.

\subsection{Sample collection and processing.}

All samples to be tested were collected at 3 weeks post each immunization.

Serum samples. Blood samples were collected by tail vein bleeding; serum was obtained by standard methods and stored at $-80^{\circ} \mathrm{C}$ until use.

Vaginal secretions. Vaginal secretions were collected by pipetting $50 \mu$ of PBS in and out of the vagina gently until a discrete clump of mucus was recovered. This usually took four to eight cycles of pipetting and required cutting the pipette tip back to a diameter of 1 to $2 \mathrm{~mm}$. A second vaginal wash with $50 \mu \mathrm{l}$ of PBS was then done to ensure more complete recovery of the vaginal secretions and it was combined with the first wash. Vaginal washes were centrifuged at $12,000 \times g$ for $10 \mathrm{~min}$ to separate the mucus from the PBS wash solution shortly after collection. The mucus and supernatant were then frozen separately at $-80^{\circ} \mathrm{C}$. The PBS wash solution contained a cocktail of proteinase inhibitors (153.8 nM Aprotinin, $3.2 \mu \mathrm{M}$ Bestatin and $10 \mu \mathrm{M}$ Leupeptin). In order to obtain a complete recovery of IgA and presumably also IgG from the vaginal mucus, samples were thawed, weighed, and extracted twice for $2 \mathrm{hr}$ each time in $100 \mu$ of PBS per sample, with rotation at $20 \mathrm{rpm}$ in a 
$12-\mathrm{ml}$ polystyrene tube at $4^{\circ} \mathrm{C}$. The two extracts and the original wash supernatant were pooled, made up to $300 \mu \mathrm{l}$ per sample, and frozen at $-80^{\circ} \mathrm{C}$ until needed $[16 ; 31]$.

Intestinal washes. Fecal pellets were resuspended at a $10 \%$ (wt/vol) concentration in a stool diluent (10 mM Tris pH 7.5, $100 \mathrm{mM} \mathrm{NaCl}, 1 \mathrm{mM} \mathrm{CaCl} 2,0.05 \%$ Tween 20, $5 \mathrm{mM}$ sodium azide, additioned with $1 \mathrm{mg}$ of aprotinin/ml and $10 \mathrm{mg}$ of leupeptin/ml). After incubation on ice for $20 \mathrm{~min}$, suspensions were centrifuged at $18,000 \mathrm{xg}$ for $15 \mathrm{~min}$ in order to remove fecal solids. Processed fecal antibody samples were stored at $-80^{\circ} \mathrm{C}[16 ; 32]$.

\subsection{Serological Assays.}

Nunclon 96 well ELISA plates were coated with $1 \mu \mathrm{g} / \mathrm{ml}$ recombinant gp160 (BioSciences, CT) or rp24 antigen (Aalto, Dublin) $0,5 \mu \mathrm{g} / \mathrm{ml}$ (both antigens of the B-clade). Serum dilutions $1 / 100-1 / 100000$ (PBS-0,5\%BSA-0,1\% Tween20) were incubated for $2 \mathrm{~h}$ at $37^{\circ} \mathrm{C}$. After 5 washes with PBS-Tween $(0.1 \%)$, wells were incubated 90 min. at $37^{\circ} \mathrm{C}$ with HRPconjugated rabbit anti-mouse IgG (BioRad) or Southern Biotech rabbit-anti-mouse IgA. After additional 5 washes with PBS-Tween (0.1\%) wells were incubated with orthophenylenediamine- $\mathrm{HCl}(\mathrm{OPD})(2 \mathrm{mg} / \mathrm{ml}$ in citrate buffer $+0.03 \% \mathrm{H} 2 \mathrm{O} 2) 30 \mathrm{~min}$. at room temperature. Absorbance was determined at $492 \mathrm{~nm}$ and reactions were considered positive when the mean absorbance of immunized animals exceeded by a factor of 3 the mean absorbance of equal dilutions of sera collected from control animals. Read abs. 492 $\mathrm{nm}$. For the fecal and vaginal washings, the ELISA was performed as above except for the primary $\mathrm{Ab}$ incubation, carried at $+4^{\circ} \mathrm{C}$ over night (approx. $16 \mathrm{hr}$ ).

\subsection{B cell epitope mapping.}

The anti-envelope serum IgG response was mapped by pooled sera using 48 individual peptides (15-mers with 5-mer overlap) representing amino acids 249-514 of gp120 HIV-1 subtype B $[33 ; 34]$. The serum was diluted in 10 -fold serial dilutions (starting with dilution 1/100) and reactive antibodies were detected with goat anti-mouse IgG antibodies 
conjugated to horseradish peroxidase (HRP) (Bio-Rad, CA, USA). Plates were developed with O-phenylenediamine and the OD was read at $490 \mathrm{~nm}$.

\subsection{HIV-1 neutralization assay.}

Serum from preimmunized and immunized mice were heat-inactivated $\left(56^{\circ} \mathrm{C}\right.$ for $30 \mathrm{~min}$ ) and serially diluted at 3 -fold dilutions, starting at $1 / 20$. Virus aliquots of the dual-tropic $T$ cell line-adapted HIV-1 SF2 strain and the primary NSI/CCR5 tropic clade A isolate 92UG031 were diluted in medium supplemented with $10 \%$ inactivated FCS (Invitrogen Life Technologies, Paisley, U.K.), 10 IU/ml IL-2 (Amersham Biosciences, Little Chalfont, U.K.), $50 \mu \mathrm{g} / \mathrm{ml}$ streptomycin, and 50 IU penicillin (Invitrogen Life Technologies). Seventy-five microliters of each virus dilution, corresponding to 40 and 80 MOI for the SF2 strain and to 30 and 60 MOI for the 92 UG031 strain, and $75 \mu$ of each serum dilution were incubated in duplicate for $1 \mathrm{~h}$ at $37^{\circ} \mathrm{C}$ in round-bottom culture plates (Nunc). Human peripheral blood mononuclear cells (PBMCs) were isolated by Ficoll-Hypaque density gradient centrifugation and seventy-five microliters containing $1 \times 10^{6} / \mathrm{ml}$ PHA-stimulated PBMC were added to each well. After $16-$ to $18-\mathrm{h}$ incubation at $37^{\circ} \mathrm{C}$, the cells were washed with RPMI 1640 , and $200 \mu \mathrm{l}$ of fresh medium was added to each well. Every 3 days, $100 \mu$ l of medium was changed. After 6-7 days, $100 \mu$ l of supernatant from each well was collected, and virus production was measured in a p24 Ag capture ELISA as previously described [35].

\subsection{Cell mediated immunity.}

Lymphocyte proliferation and cytokine production. The lymphocyte proliferation was performed essentially as described earlier [36]. Single-cell suspensions were prepared from spleen and lymph nodes and $4 \times 10^{5}$ cells/per well were co-cultured with antigen $(0.1 \mathrm{mg}$ rgp160 or rgagp24 or medium, ProteinSciences, CT per well) for 3 days before tritium-H3labeled thymidine $(1 \mathrm{uCi} / \mathrm{ml})$ was added to each well and incubated for $16 \mathrm{~h}$. The cells were harvested and counted in a $\beta$-counter (Wallac1450, Finland). After $72 \mathrm{~h}$, interferon- $\gamma$ was 
determined in the culture supernatants by ELISA in accordance with the manufacturer's instructions (R\&DSystems, UK).

\subsection{Statistical analyses.}

Intergroup comparisons were performed with the unpaired two-sided Student's $t$-test. All $P$ values were two-tailed and considered significant if less than 0.05 . 


\section{RESULTS}

\subsection{Induction of serum antibody response by intra-nasal administration.}

HIV-VLP $\mathrm{A}_{\mathrm{S}}$, produced in insect cells and purified on a $10-60 \%$ continuous sucrose gradient [11], were administered intra-nasally in Balb/c mice following a prime-boost protocol defined "homologous" (VLP prime + VLP boost) or "heterologous" (DNA prime + VLP boost) boosting, with or without the $\mathrm{L} 3$ adjuvant (Table 1 ). Moreover, to achieve a broader neutralizing activity, the heterologous protocol can be considered also a multiclade approach, given that the DNA plasmid expresses a B-clade and the VLP an A-clade envelope.

Animals were boosted 4 weeks after the priming immunization and serum was collected by tail vein bleeding three weeks after the single boosting dose. Each immunization group contained 6 animals which did not show signs of toxicity and remained healthy up to the end of the vaccination protocol.

ELISA tests were performed on microwell plates coated with recombinant gp160 or p24. The data show that the heterologous prime-boost protocol induces a statistically significant increase of serum anti-env IgG and IgA titers, compared to the homologous protocol. Only the IgA titers are further increased in the heterologous protocol when the single VLP boost is adjuvanted in L3 (Fig. 1A and C). The serum anti-gag IgG and IgA titers, instead, are low and equivalent in both homologous and heterologous nonadjuvanted protocols, resulting considerably increased (1 - 2 logs) in both protocols when the single VLP boost is adjuvanted in L3 (Fig. 1B and D).

\subsection{Induction of mucosal antibody response by intra-nasal administration.}

In order to quantify the antibody response at mucosal level, vaginal and intestinal washes were collected from each animal three weeks after the single VLP boosting dose and evaluated in the ELISA system. The results show that, also at vaginal as well as intestinal level, the heterologous prime-boost protocol induces a statistically significant increase of anti-env IgG and IgA titers, compared to the homologous protocol. Only the 
intestinal IgA titers are further increased in the homologous protocol when the single VLP boost is adjuvanted in L3 (Fig. 2A and C).

A
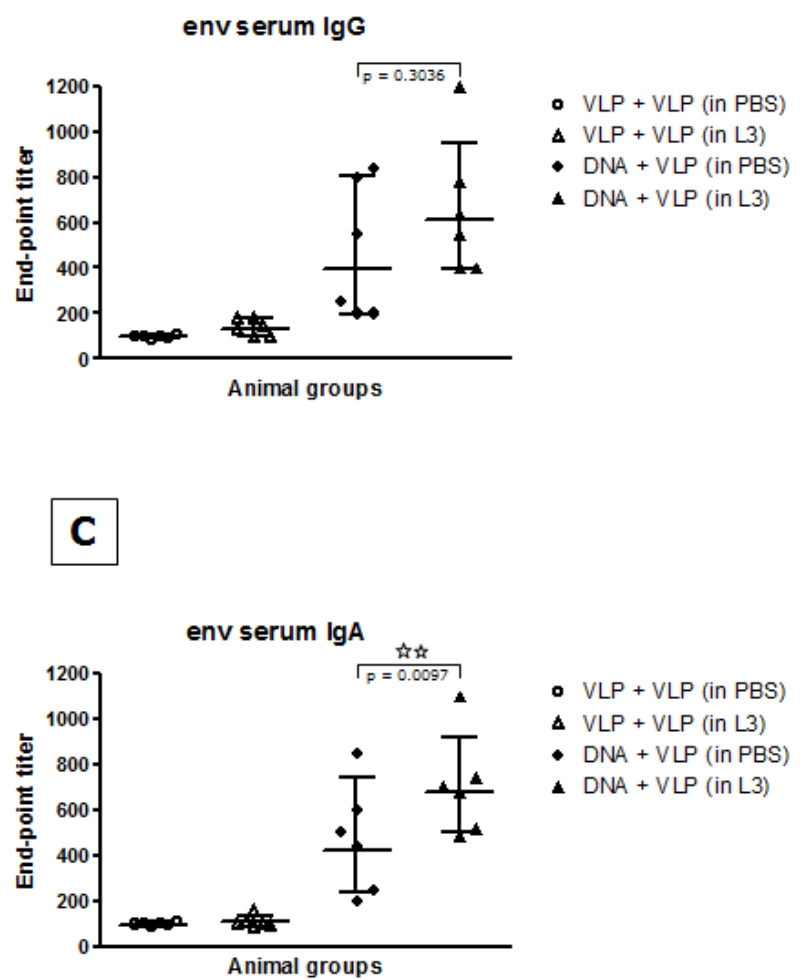

\section{B}

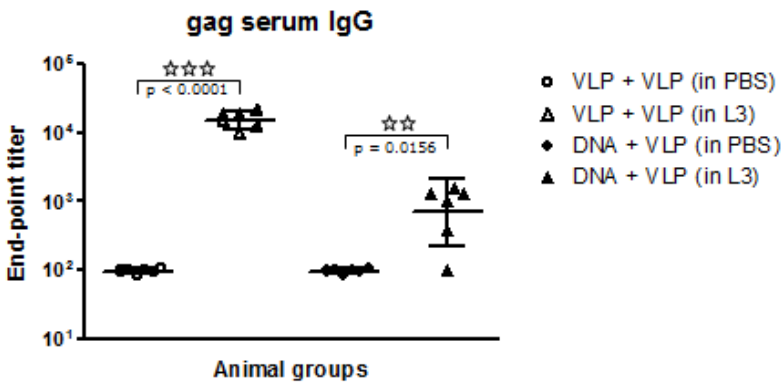

D

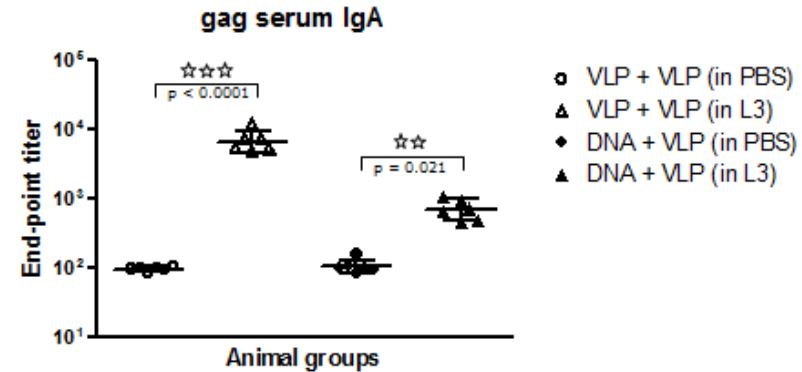

Fig. 1 Levels of humoral response induced in mice. Serum samples were obtained at the end of the immunization protocol and analyzed for specific IgG ( $A$ and $B$ ) and IgA ( $C$ and $D$ ) antibodies by ELISA, performed in triplicate on 96-well plates coated with either B-clade rgp160 or rp24. Results are expressed as the individual average reciprocal last dilution with a threefold O.D.492 of the pre-immune sera. Geometric mean titers with $95 \%$ confidence interval are indicated for each animal group. $P$ values are indicated for intergroup comparisons and stars indicate significant difference between compared groups.

The mucosal anti-gag IgA titers are low and equivalent in both homologous and heterologous non-adjuvanted protocols, resulting significantly increased in both protocols when the single VLP boost is adjuvanted (Fig. 2B and D). The magnitude of this increase is particularly relevant at vaginal level, calculated as a 26.6 -fold increase of the geometric mean titer in the homologous protocol and 47.9-fold increase in the heterologous protocol.

The results obtained at systemic and mucosal levels with these prime-boost protocols, performed with a single VLP boosting dose of 2ug, are particularly interesting compared to 
A

env vaginal IgA

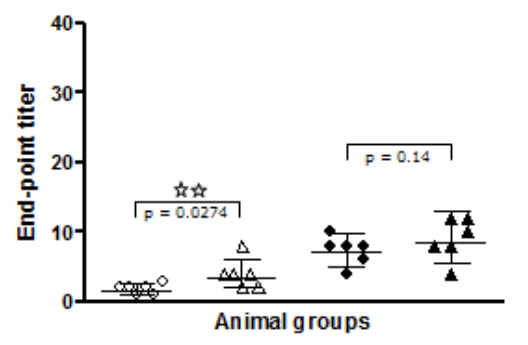

C

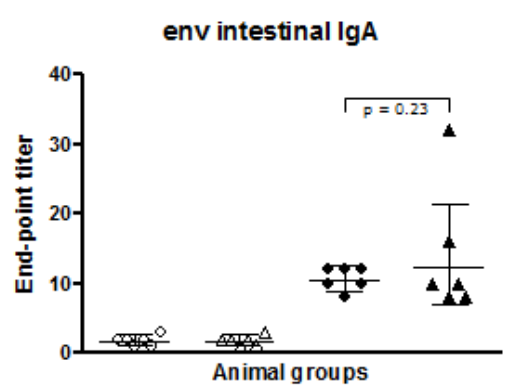

B

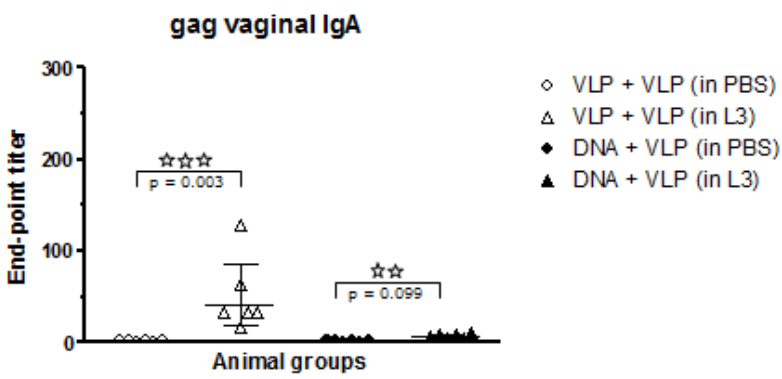

D

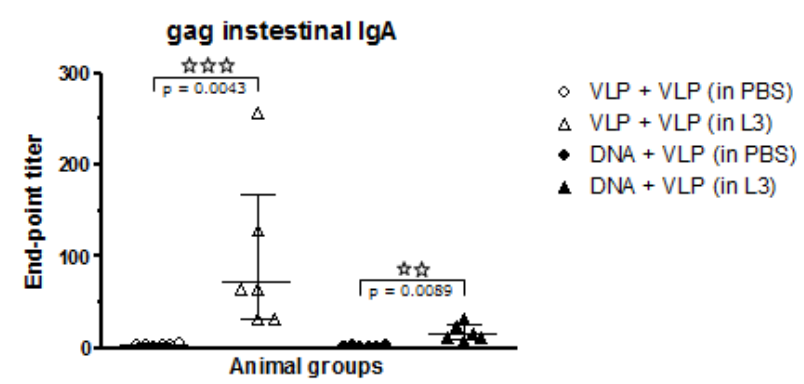

Fig. 2 Levels of antibodies at mucosal (vaginal and intestinal) sites. Vaginal and intestinal washes were obtained at the end of the immunization protocol from each animal. Levels of specific IgA antibodies at vaginal (A and B) and intestinal (C and D) sites were evaluated by ELISA, performed in triplicate on 96-well plates coated with either rgp160 or rp24. Results are expressed as the individual average reciprocal last dilution with a threefold O.D.492 of the pre-immune sera. Geometric mean titers with $95 \%$ confidence interval are indicated for each animal group. $P$ values are indicated for intergroup comparisons and stars indicate significant difference between compared groups.

our previous results obtained with 4 doses of VLP (20 and $100 \mathrm{ug} / \mathrm{dose}$ ) without adjuvant [16]. In particular, the vaginal IgA anti-gag response is considerably increased in both homologous and heterologous protocols adjuvanted in L3 (8.06 folds the homologous and 14.38 folds the heterologous) (Table 2). At intestinal level, the homologous protocol does not improve the previously observed response, while the heterologous protocol induces a 2-3-fold increase of IgA titer compared to previous immunization schedules as well as the homologous protocol (Table 2).

The amounts of fecal and vaginal washings were not significantly different between the study groups. The mean and SD amounts of total IgA for the four 
fecal IgA 64+/- 8,4 ug/ml, vaginal IgA $15+/-8,7$ ug/ml, Group VLP+VLP (in L3)

fecal IgA 91,7 +/- 12,7 ug/ml, vaginal $13+/-4$ ug/ml, Group DNA+VLP (in PBS)

fecal IgA $79+/-4,5$ ug/ml, vaginal IgA 14,5+/- 6 ug/ml and Group DNA+VLP (in

L3) fecal IgA 69+/- 11 ug/ml, vaginal IgA 11+/- 3 ug/ml.

\subsection{Evaluation of B-cell epitopes recognized by immune sera.}

Serum from immunized animals were evaluated for mapping their recognition of B cell epitopes in the gp120 C2 - V5 region, using overlapping linear peptides as target. Serum from animals immunized with either the homologous or the heterologous prime-boost protocol showed a wide-spread recognition pattern along the considered region (Fig. 3 ). In particular, the DNA+VLP heterologous protocol mostly induced an increased binding to individual epitopes covering the C2-V3 region, compared to the VLP-VLP homologous protocol, and did not present additional "masked" epitopes to the immune system. On the contrary, the two protocols induced either the same level of recognition or very limited response against other epitopes (Fig. 3). Overall, the strongest binding was observed against the V3 and V4 peptides, outside the gp120 regions identified as involved in the CD4 binding [37].

\subsection{Ex vivo neutralization activity of immune sera.}

Neutralization activity of antibodies induced by the single HIV-VLP $\mathrm{A}_{\mathrm{S}}$ boost in mice immunized with either the homologous or the heterologous prime-boost protocol, was verified ex vivo against heterologous $A$ - and $B$-clade isolates. The results showed that immune sera derived from mice immunized with both non-adjuvanted protocols, did not show a significant neutralization activity against the virus isolates (data not shown). On the contrary, when the single VLP boosting dose was administered in the L3 adjuvant, the immune sera induced by the homologous protocol show a $>50 \%$ neutralization activity at a $1: 20$ final dilution against both $A$ and $B$ heterologous isolates, when used at a MOI of 30 and 40, respectively (Fig. $4 \mathrm{~A}$ and $\mathrm{C}$ ). The neutralization activity was not as efficient when 


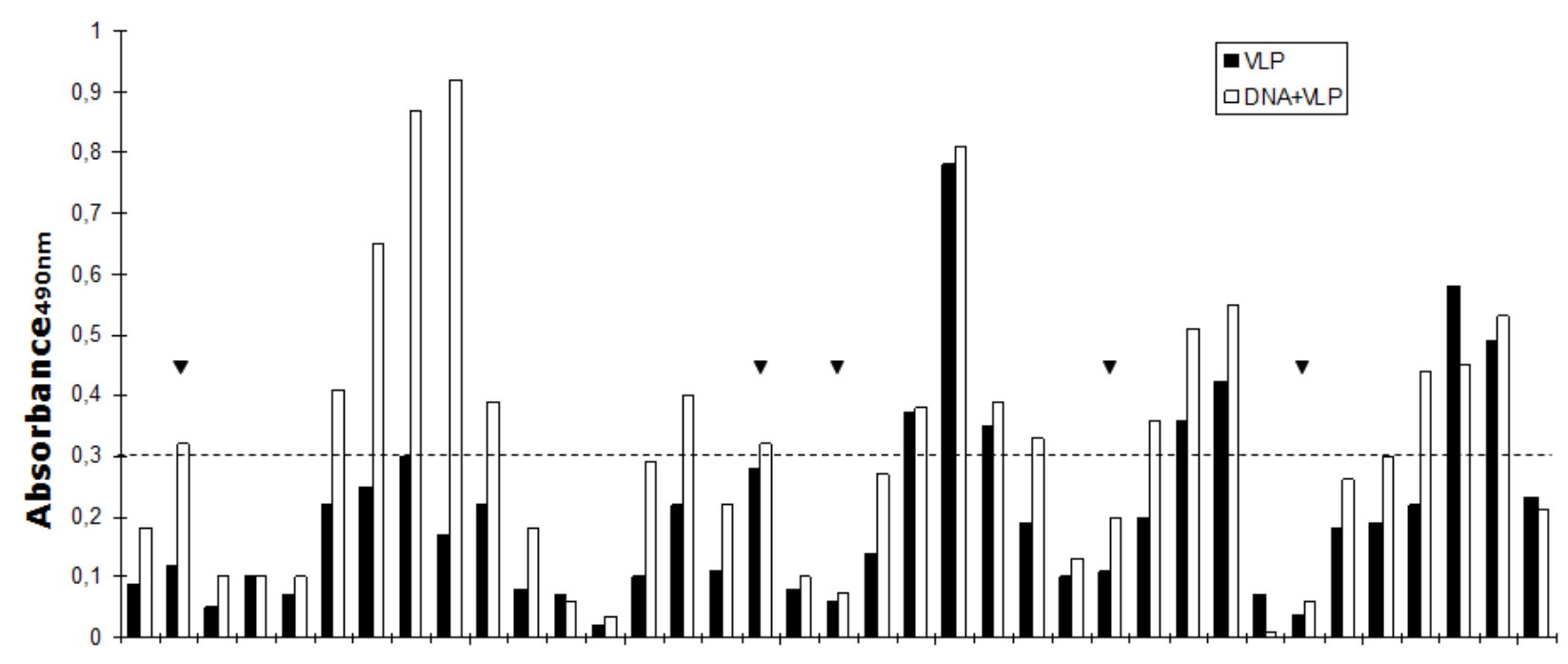
C2
$1 / 3$
C3
144
C4
155.65

Fig. 3 B cell epitope mapping. Sera from each group of animals were screened against individual 15-mer peptides covering the gp120 C2-C5 region. The assay was run at dilution 1:100 with a naïve serum cutoff of 0.3 (dotted line). Triangles indicate gp120 CD4 binding regions.

the heterologous isolates were used at a double MOI (Fig. 4 B and D). The immune sera induced by the heterologous protocol, instead, showed a $>50 \%$ neutralization activity up to a 1:60 final dilution against the heterologous B-clade isolate, at either MOI (Fig. $4 \mathrm{E}$ and F). A $>50 \%$ neutralization activity was observed at a $1: 20$ final dilution against the heterologous primary A-clade isolate, only when used at a 30 MOI (Fig. $4 \mathrm{G}$ and H).

The pre-immune sera from immunized mice did not show any neutralization activity and both viral isolates showed a similar effective kinetic of replication in the 7-day p.i. experiment, characterized by a progressive increase of virus production, measured as p24 concentration (data not shown).

\subsection{Cellular response in HIV-VLPA ${ }_{A}$-immunized animals.}

To assess the induction of Env- and Gag-specific cellular response, spleens and lymph nodes were removed from immunized mice and the IFN $\gamma$ production was evaluated in cell culture after ex vivo restimulation with either Env or Gag peptides. 

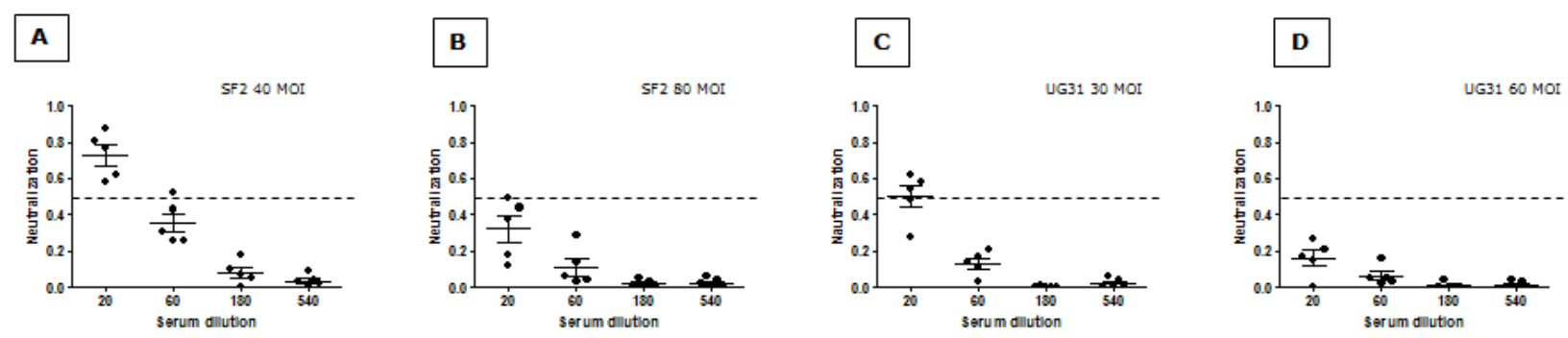

DNA + VLP in Eurocine L3
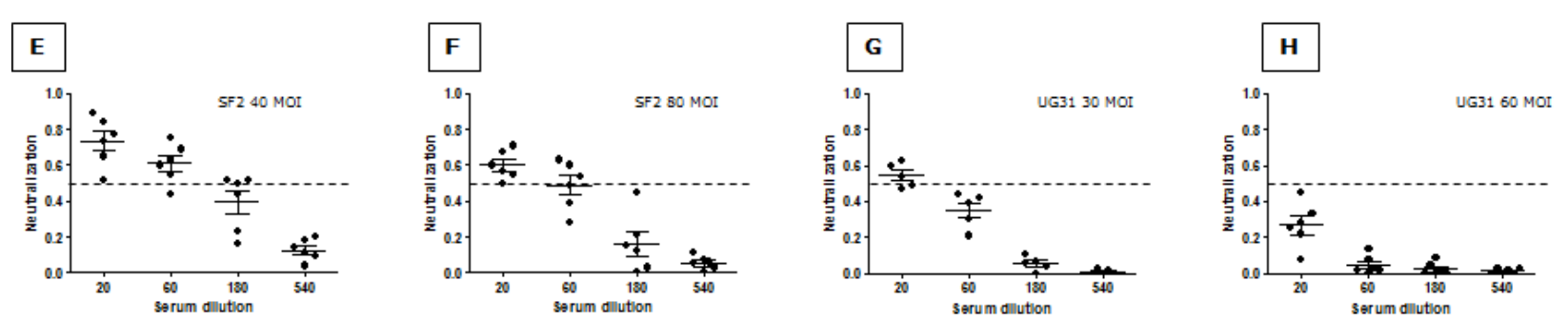

Fig. 4 Ex vivo heterologous neutralization analysis. The HIV-1 neutralization assay was performed on PHA-stimulated PBMCs using two heterologous $A$ and $B$ viral isolates. Two different TCID 50 of each isolate (40 and 80 for SF2; 30 and 60 for 92UG031) were pretreated with serial dilutions of the immune sera, collected at the end of the complete immunization schedule. The individual neutralization activity of serum antibodies, at different dilutions, is represented as percentage of the virus replication compared to control samples. Mean titers \pm SEM are indicated for each animal group. 50\% neutralization (dotted line) has been considered the lowest limit to score positive the test.

The results showed that the heterologous prime-boost protocol induces a statistically significant increase of increased IFN $\gamma$ production specific for the Env epitopes in the spleen and lymph nodes, compared to the homologous protocol. This is not further increased in the both protocols when the single VLP boost is adjuvanted in L3 (Fig. 5A and C). The IFN $\gamma$ production specific for the Gag epitopes in the spleen and lymph nodes, instead, are low and equivalent in both homologous and heterologous non-adjuvanted protocols, resulting considerably increased in both protocols when the single VLP boost is adjuvanted in L3 (Fig. 5B and D). 
A

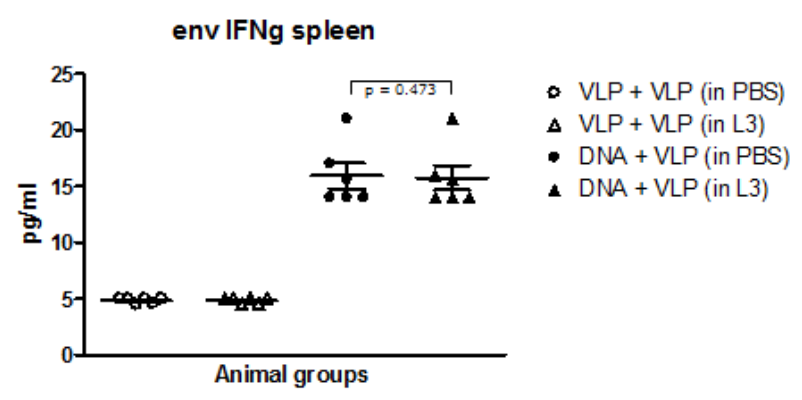

$\mathbf{C}$

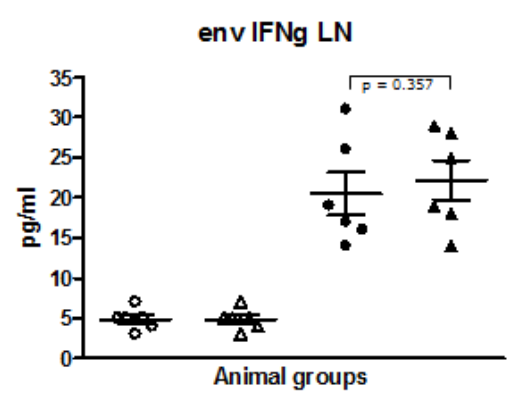

B

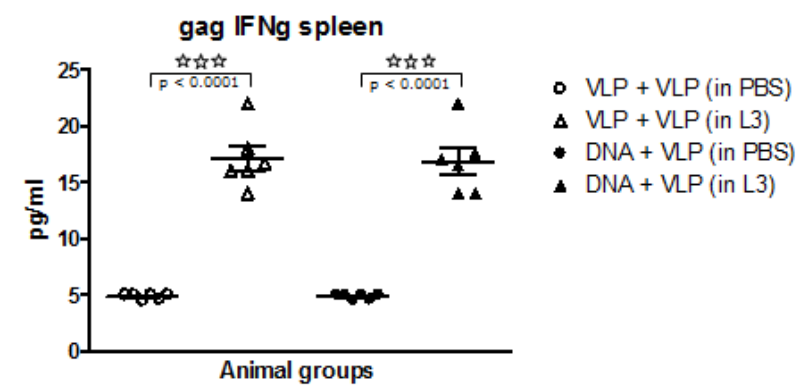

D

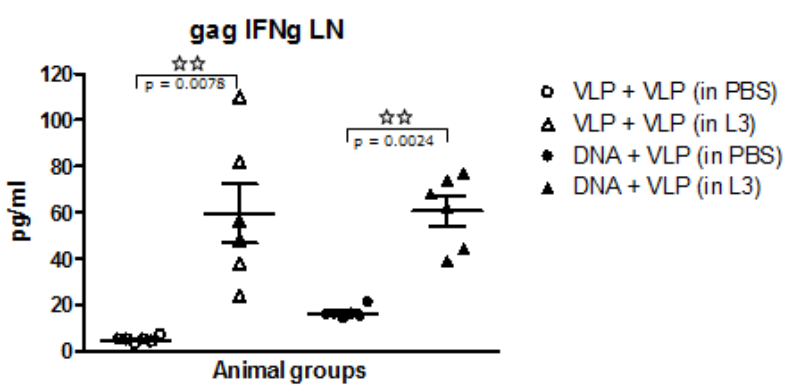

Fig. 5 Cellular immunity: IFN $\gamma$ production. Spleen and lymph node cells from immunized mice were isolated and cultured for 3 days in the presence of Env or Gag peptides. Results are represented as the IFN $\gamma$ produced by individual animals and mean titers \pm SEM are indicated for each animal group. $P$ values are indicated for intergroup comparisons and stars indicate significant difference between compared groups.

In particular, the levels of anti-gag IFN $\gamma$ production were significantly higher in the lymph node than in the spleen $(p<0.0001)$.

Similar results were observed evaluating the proliferation of $\mathrm{T}$ helper cells from immunized mice when ex vivo restimulated with either Env or Gag peptides. Again, the heterologous prime-boost protocol induces a statistically significant increase of T helper proliferation (expressed as Stimulation Index - S.I.) specific for the Env epitopes, compared to the homologous protocol. This is not further increased in the both protocols when the single VLP boost is adjuvanted in L3 (Fig. 6A). The T helper proliferation specific for the Gag epitopes, instead, are low and equivalent in both homologous and heterologous non-adjuvanted protocols, resulting statistically increased in both protocols when the single VLP boost is adjuvanted in L3 (Fig. 6B). 


\section{A}

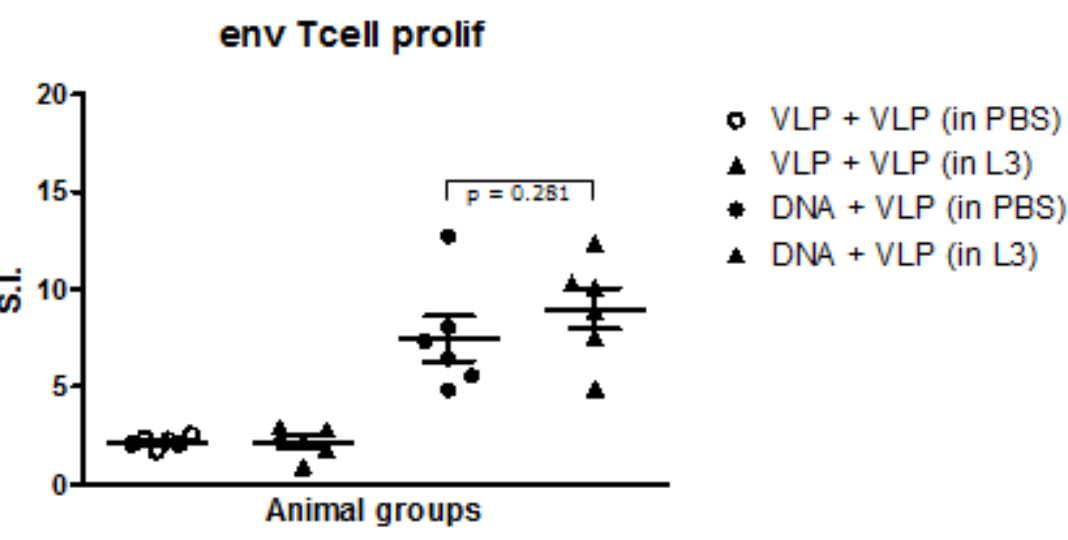

B

gag Tcell prolif

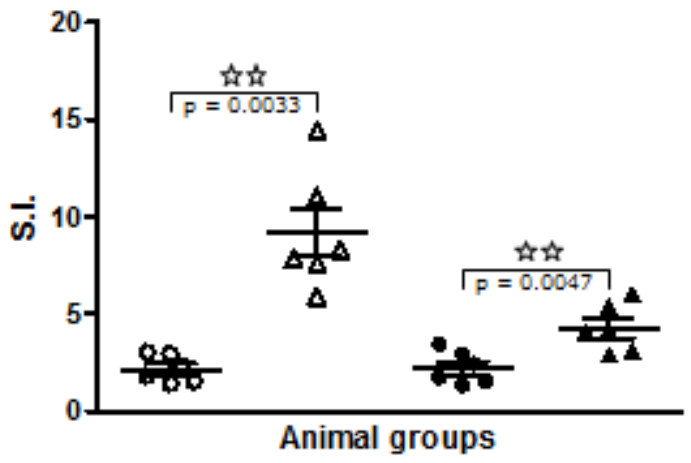

- VLP + VLP (in PBS)

$\triangle \mathrm{VLP}+\mathrm{VLP}$ (in L3)

- DNA + VLP (in PBS)

- DNA + VLP (in L3)

Fig. 6 Cellular immunity: T helper proliferation. Spleen cells from immunized mice were isolated and cultured for 3 days in the presence of Env or Gag peptides. The Stimulation Index (S.I.) represents the fold increase of thymidine incorporation in cells from immunized animals compared to cells from control animals. Results are represented as the S.I. identified in individual animals and mean titers \pm SEM are indicated for each animal group. $P$ values are indicated for intergroup comparisons and stars indicate significant difference between compared groups. 


\section{DISCUSSION}

We have previously reported that a candidate HIV-1 preventive vaccine, based on VLPS expressing a gp120 glycoprotein derived from an HIV-1 clade A isolate (HIV-VLPAS) [11], induces IgA and/or IgG antibody responses in sera as well as at mucosal (vaginal and intestinal) sites and CTL activity when administered by multi-regimen protocol in BALB/C mice by the intra-nasal route in the absence of adjuvants $[12 ; 16]$. These results were obtained using 20 and $100 \mu \mathrm{g} /$ dose.

In the present study, the HIV-VLPA $\mathrm{S}$ immunogenicity has been evaluated in a Balb/c mouse model by intra-nasal administration, comparing a VLP+VLP homologous vs a DNA+VLP heterologous prime-boost immunization protocol and evaluating the enhancing effect of the Eurocine L3 mucosal adjuvant. In this case, the single VLP boosting dose was $2 \mu \mathrm{g}$.

The results showed that the non-adjuvanted heterologous protocol induced a statistically significant increase of serum anti-env Ig titers, compared to the homologous protocol, and that the single adjuvanted HIV-VLPA boost, either in the homologous or in the heterologous protocol, induced a relevant increase of the serum anti-gag response. This effect was less dramatic for the anti-env response but a statistically significant increase of serum IgA was observed in the heterologous protocol (Fig. 1 A-D). Similar results were observed at mucosal (vaginal and intestinal) sites (Fig. 2 A-D).

The B-cell epitope mapping analysis showed that the homologous and the heterologous prime-boost protocols are comparable in presenting gp120 epitopes to the immune system, given that no epitope appears to be exposed only in one of the two protocols (Fig. 3). The increased antibody levels to the V3 epitopes in the DNA+VLP heterologous protocol may result from the higher env antigen load and/or a different presentation associated with the DNA administration. On the other hand, equivalent levels of response to other peptides between the two protocols would suggest a more efficient presentation of the conserved cross-clade epitopes by the HIV-VLPAS. It remains to be further investigated whether the 
strong binding to linear peptides outside the identified CD4-binding regions plays a role in the neutralization activity of induced antibodies.

Serum from mice immunized with the homologous and heterologous prime-boost protocols showed a $>50 \%$ neutralizing activity against heterologous $A$ and $B$ clade viral isolates. In particular, the heterologous boosting protocol resulted more efficient in inducing a neutralizing activity against the heterologous B-clade isolate, likely for the higher B-clade env antigen load associated to the DNA administration. Nevertheless, a significant cross-clade neutralizing activity was observed, also in the homologous boosting protocol, confirming our previous observations $[12 ; 16]$. Additional improvements of the observed cross-clade neutralizing efficacy should be obtained by multiple VLP booster doses.

Furthermore, the non-adjuvanted heterologous protocol induced a statistically significant increase of IFN $\gamma$ production (Fig. $5 \mathrm{~A}$ and C) and T helper proliferation (Fig. $6 \mathrm{~A}$ ) specific for env epitopes, compared to the homologous protocol, not further increased in the both protocols when the single VLP boost is adjuvanted. The single adjuvanted HIV$\mathrm{VLP}_{\mathrm{A}}$ boost, either in the homologous or in the heterologous protocol, induced an increased IFN $\gamma$ production (Fig. 5 B and D) and T helper proliferation (Fig. 6 B) specific for gag epitopes, compared to the protocols including a non-adjuvanted VLP.

These data confirm the efficient induction of humoral and cellular systemic as well as mucosal immunity by intra-nasal administration of HIV-VLPA $\mathrm{S}$, as previously reported [16]. This is likely due to efficient presentation in the major histocompatibility complex (MHC) class I and II context with a resulting activation of both Th1 and Th2 pathways, as recently shown in studies on VLP-activated dendritic cells $[38 ; 39]$.

The designed heterologous protocol, based on a DNA prime and a single VLP boosting dose, is able to increase the env-specific humoral and cellular immune response, which is to some extent increased by the administration of adjuvanted VLP boost. On the contrary, 
the L3 adjuvant appears to be extremely efficient in increasing the anti-gag response in both homologous and heterologous protocol.

These results are obtained with a $1 / 10$ of the previously used HIV-VLPAS dose ( 2 vs 20 $\mu \mathrm{g})$, inducing significantly higher geometric mean titers of systemic and mucosal antibodies, with cross-clade neutralizing activity, compared to our previous studies performed with multiple doses of non-adjuvanted VLPS $[12 ; 16]$. This strongly indicates that the prime boost protocols and, in particular, the adjuvant effect of the L3 is extremely efficient in stimulating the mucosa-associated lymphoid tissue, possibly initiating and supporting the transition from innate to adaptive immunity. The results confirm previous observations reported for the heat-killed bacille Calmette-Guerin (BCG) model which, formulated in the L3 adjuvant, induces immune response and immune protection comparable to the conventional live BCG vaccine [29].

The more efficient induction of anti-gag than ant-env response with a single VLP boost, either in the homologous or in the heterologous protocol, strongly suggests that the Gag:Env ratio in the HIV-VLPAS is in great favor of Gag, as described for the native HIV-1 particles (40 to $60: 1$, Gag:Env) $[40 ; 41]$. This implies that the antigen load carried by the HIV-VLP ${ }_{A} S$ is significantly higher for gag than for env and, consequently, the single HIV$\operatorname{VLP}_{\mathrm{A}}$ dose is more efficient in boosting the anti-gag response. Moreover, this observation could also result from the generally reported lower immunogenicity of env compared to gag protein, as consequence of high degree of protein glycosylation $[42 ; 43]$. To ensure an optimal env antigen presentation, immunization protocols designed with multiple HIV-VLPA boosting doses are under evaluation.

In particular, the relevant anti-gag humoral response, not expected to be involved in the classic viral neutralization, is of high relevance given the several reports showing the role of non-neutralizing anti-gag antibodies in the protection/containment of the HIV-1 infection [44-49]. 
The A and B multi-clade composition of the described strategy results in an efficient ex vivo cross-clade neutralization activity which can be further improved by additional VLP boosting doses. These results suggest a possible broad in vivo protection which would make this strategy a good candidate for a vaccine approach targeted to sub-Saharan countries, with high incidences of HIV-1 clade A isolates, as well as to Countries characterized by multiclade HIV-1 epidemics.

\section{ACKNOWLEDGMENTS}

This study was supported by grants from the Ministero Italiano della Sanita` (Ricerca Corrente and Progetto Finalizzato AIDS 2000) and the ICSC-World Lab, Lausanne, Switzerland (project MCD-2/7). The study (Jorma Hinkula and Britta Wahren) was further supported by grants from the Swedish Research Council and Swedish Medical Society. 
Reference List

[1] Kirnbauer R, Booy F, Cheng N, Lowy DR, Schiller JT. Papillomavirus L1 major capsid protein self-assembles into virus- like particles that are highly immunogenic. Proc Natl Acad Sci USA 1992;89:12180-4.

[2] Garnier L, Ravallec M, Blanchard P, et al. Incorporation of pseudorabies virus gD into human immunodeficiency virus type $1 \mathrm{gag}$ particles produced in baculovirus-infected cells. J Virol 1995;69:4060-8.

[3] Li TC, Yamakawa Y, Suzuki K, et al. Expression and self-assembly of empty virus-like particles of hepatitis E virus. J Virol 1997;71:7207-13.

[4] O'Neal CM, Crawford SE, Estes MK, Conner ME. Rotavirus Virus-Like Particles administered mucosally induce protective immunity. J Virol 1997;71:8707-17.

[5] Sedlik C, Saron M-F, Sarraseca J, Casal I, Leclerc C. Recombinant parvovirus-like particles as an antigen carrier: a novel nonreplicative exogenous antigen to elicit protective antiviral cytotoxic T cells. Proc Natl Acad Sci U S A 1997;94:7503-8.

[6] Guerrero RA, Ball JM, Krater SS, Pacheco SE, Clements JD, Estes MK. Recombinant Norwalk virus-like particles administered intranasally to mice induce systemic and mucosa (fecal and vaginal) immune responses. J Virol 2001;75:9713-22.

[7] Gheysen D, Jacobs E, de Foresta F, et al. Assembly and release of HIV-1 precursor Pr55 $5^{\text {gag }}$ virus-like particles from recombinant baculovirus-infected insect cells. Cell $1989 ; 59: 103-12$.

[8] Rovinski B, Haynes JR, Cao SX, et al. Expression and characterization of genetically engineered human immunodeficiency virus-like particles containing modified envelope glycoproteins: implications for development of a cross- protective AIDS vaccine. J Virol 1992;66:4003-12.

[9] Griffiths JC, Harris SJ, Layton GT, et al. Hybrid human immunodeficiency virus Gag particles as an antigen carrier system: Induction of cytotoxic T-cell and humoral responses by a Gag:V3 fusion. J Virol 1993;67:3191-8.

[10] Deml L, Schirmbeck R, Reimann J, Wolf H, Wagner R. Recombinant human immunodeficiency Pr55 $5^{\text {gag }}$ virus-like particles presenting chimeric envelope glycoproteins induce cytotoxic T-cells and neutralizing antibodies. Virology 1997;235:26-39.

[11] Buonaguro L, Buonaguro FM, Tornesello ML, et al. High efficient production of Pr55gag Virus-like Particles expressing multiple HIV-1 epitopes, including a gp120 protein derived from an Ugandan HIV-1 isolate of subtype A. Antiviral Research 2001;49:35-47.

[12] Buonaguro L, Racioppi $L$, Tornesello $M L$, et al. Induction of neutralizing antibodies and CTLs in Balb/c mice immunized with Virus-like Particles presenting a gp120 molecule from a HIV-1 isolate of clade A (HIV-VLPAs). Antiviral Research 2002;54:189-201.

[13] Young KR, McBurney SP, Karkhanis LU, Ross TM. Virus-like particles: designing an effective AIDS vaccine. Methods 2006;40:98-117. 
[14] Buonaguro L, Del Gaudio E, Monaco M, et al. Heteroduplex mobility assay and phylogenetic analysis of V3 region sequences of HIV 1 isolates from Gulu - Northern Uganda. J Virol 1995;69:7971-81.

[15] Buonaguro L, Buonaguro FM, Russo F, et al. A novel gp120 sequence from an HIV-1 isolate of the A clade identified in North Uganda. AIDS Res Hum Retroviruses $1998 ; 14: 1287-9$.

[16] Buonaguro L, Visciano ML, Tornesello ML, Tagliamonte M, Biryahwaho B, Buonaguro FM. Induction of systemic and mucosal cross-clade neutralizing antibodies in BALB/C mice immunized with human immunodeficiency virus type 1 clade $A$ virus-like particles administered by different routes of inoculation. J Virol 2005;79:7059-67.

[17] Schneider J, Gilbert SC, Hannan CM, et al. Induction of CD8+ T cells using heterologous prime-boost immunisation strategies. Immunol Rev 1999;170:29-38.

[18] Ramshaw IA, Ramsay AJ. The prime-boost strategy: exciting prospects for improved vaccination. Immunol Today $2000 ; 21: 163-5$.

[19] Zolla-Pazner S. Identifying epitopes of HIV-1 that induce protective antibodies. Nat Rev Immunol 2004;4:199-210.

[20] Humbert M, Dietrich U. The role of neutralizing antibodies in HIV infection 17. AIDS Rev 2006;8:51-9.

[21] Mc Cann CM, Song RJ, Ruprecht RM. Antibodies: can they protect against HIV infection? Curr Drug Targets Infect Disord 2005;5:95-111.

[22] Srivastava IK, Ulmer JB, Barnett SW. Role of neutralizing antibodies in protective immunity against HIV. Hum Vaccin 2005;1:45-60.

[23] Shu Y, Winfrey S, Yang ZY, et al. Efficient protein boosting after plasmid DNA or recombinant adenovirus immunization with HIV-1 vaccine constructs. Vaccine 2007; $25: 1398-408$.

[24] Rasmussen RA, Ong $\mathrm{H}$, Kittel $\mathrm{C}$, et al. DNA prime/protein boost immunization against HIV clade C: safety and immunogenicity in mice. Vaccine $2006 ; 24: 2324-32$.

[25] Girard M, Osmanov SK, Kieny MP. A review of vaccine research and development: the human immunodeficiency virus (HIV). Vaccine 2006;24:4062-81.

[26] Montefiori DC, Safrit JT, Lydy SL, et al. Induction of neutralizing antibodies and gagspecific cellular immune responses to an R5 primary isolate of human immunodeficiency virus type 1 in rhesus macaques. J Virol 2001;75:5879-90.

[27] Radaelli A, Zanotto C, Perletti G, et al. Comparative analysis of immune responses and cytokine profiles elicited in rabbits by the combined use of recombinant fowlpox viruses, plasmids and virus-like particles in prime-boost vaccination protocols against SHIV. Vaccine 2003;21:2052-64.

[28] Schroder U, Svenson SB. Nasal and parenteral immunizations with diphtheria toxoid using monoglyceride/fatty acid lipid suspensions as adjuvants. Vaccine 1999; 17:2096-103. 
[29] Haile M, Schroder U, Hamasur B, et al. Immunization with heat-killed Mycobacterium bovis bacille Calmette-Guerin (BCG) in Eurocine L3 adjuvant protects against tuberculosis. Vaccine 2004;22:1498-508.

[30] Hinkula J, Devito C, Zuber B, et al. A novel DNA adjuvant, N3, enhances mucosal and systemic immune responses induced by HIV-1 DNA and peptide immunizations. Vaccine 2005; Epub, sept 6.

[31] Parr EL, Parr MB. Immunoglobulin G, plasma cells, and lymphocytes in the murine vagina after vaginal or parenteral immunization with attenuated Herpes Simplex Virus type 2. J Virol 1998;72:5137-45.

[32] VanCott TC, Kaminski RW, Mascola JR, et al. HIV-1 neutralizing antibodies in the genital and respiratory tracts of mice intranasally immunized with oligomeric gp 160 . J Immunol 1998;160:2000-12.

[33] Rollman E, Hinkula J, Arteaga J, et al. Multi-subtype gp160 DNA immunization induces broadly neutralizing anti-HIV antibodies. Gene Ther 2004;11:1146-54.

[34] Broliden PA, von GA, Clapham P, et al. Identification of human neutralizationinducing regions of the human immunodeficiency virus type 1 envelope glycoproteins. Proc Natl Acad Sci U S A 1992;89:461-5.

[35] Devito C, Levi M, Broliden K, Hinkula J. Mapping of B-cell epitopes in rabbits immunised with various gag antigens for the production of HIV-1 gag capture ELISA reagents. J Immunol Methods 2000;238:69-80.

[36] Ljungberg K, Rollman E, Eriksson L, Hinkula J, Wahren B. Enhanced immune responses after DNA vaccination with combined envelope genes from different HIV-1 subtypes. Virology 2002;302:44-57.

[37] Olshevsky U, Helseth E, Furman C, Li J, Haseltine W, Sodroski J. Identification of individual human immunodeficiency virus type 1 gp120 amino acids important for CD4 receptor binding. J Virol 1990;64:5701-7.

[38] Arico E, Wang $E$, Tornesello $M L$, et al. Immature monocyte derived dendritic cells gene expression profile in response to Virus-Like Particles stimulation. J Transl Med $2005 ; 3: 45$.

[39] Buonaguro L, Tornesello ML, Tagliamonte $M$, et al. Baculovirus-derived human immunodeficiency virus type 1 virus-like particles activate dendritic cells and induce ex vivo T-cell responses. J Virol 2006;80:9134-43.

[40] Layne SP, Merges MJ, Dembo M, et al. Factors underlying spontaneous inactivation and susceptibility to neutralization of human immunodeficiency virus. Virology 1992; 189:695-714.

[41] Chertova E, Bess JJ, Jr., Crise BJ, et al. Envelope glycoprotein incorporation, not shedding of surface envelope glycoprotein (gp120/SU), Is the primary determinant of SU content of purified human immunodeficiency virus type 1 and simian immunodeficiency virus. J Virol 2002;76:5315-25.

[42] Reitter JN, Means RE, Desrosiers RC. A role for carbohydrates in immune evasion in AIDS. Nat Med 1998;4:679-84. 
[43] Cole KS, Steckbeck JD, Rowles JL, Desrosiers RC, Montelaro RC. Removal of N-linked glycosylation sites in the $\mathrm{V} 1$ region of simian immunodeficiency virus gp120 results in redirection of B-cell responses to V3. J Virol 2004; 78:1525-39.

[44] Miller C], Genesca M, Abel K, et al. Antiviral antibodies are necessary for control of SIV replication. J Virol 2007.

[45] Montefiori DC. Role of complement and FC receptors in the pathogenesis of HIV-1 infection. Springer Semin Immunopathol 1997;18:371-90.

[46] Hirsch VM, Fuerst TR, Sutter G, et al. Patterns of viral replication correlate with outcome in simian immunodeficiency virus (SIV)-infected macaques: effect of prior immunization with a trivalent SIV vaccine in modified vaccinia virus Ankara. J Virol $1996 ; 70: 3741-52$.

[47] Binley JM, Klasse PJ, Cao Y, et al. Differential regulation of the antibody responses to Gag and Env proteins of human immunodeficiency virus type 1. J Virol $1997 ; 71: 2799-809$.

[48] Forster SM, Osborne LM, Cheingsong-Popov R, et al. Decline of anti-p24 antibody precedes antigenaemia as correlate of prognosis in HIV-1 infection. AIDS $1987 ; 1: 235-40$.

[49] Weber JN, Clapham PR, Weiss RA, et al. Human immunodeficiency virus infection in two cohorts of homosexual men: neutralising sera and association of anti-gag antibody with prognosis. Lancet 1987;1:119-22. 
Table 1. Immunization scheme. The VLP dose was of $2 \mu \mathrm{g}$, the DNA plasmid dose was of $8 \mu \mathrm{g}$. The boosting dose was administered 4 weeks after the priming dose. Each group consisted of 6 animals.

\begin{tabular}{|c|c|c|}
\hline Group & Prime & Boost \\
\hline $\mathbf{1}$ & VLP (PBS) & VLP (PBS) \\
\hline $\mathbf{2}$ & VLP (L3) & VLP (L3) \\
\hline $\mathbf{3}$ & DNA (N3) & VLP (PBS) \\
\hline 4 & DNA (N3) & VLP (L3) \\
\hline
\end{tabular}

Table 2. Comparison of systemic and mucosal humoral responses induced by HIV-VLP $\mathbf{A}_{\mathrm{A}}$ in different immunization protocols. Geometric mean titers (GMT) are shown per each group. Bold and italics characters indicate a GMT 2-fold greater, or more, than the one observed in our previous study.

\begin{tabular}{|c|c|c|c|c|c|c|c|c|c|c|}
\hline & VLP 20ug* $^{*}$ & \multirow{2}{*}{ VLP 100ug* $^{*}$} & \multicolumn{2}{|c|}{ VLP+VLP } & \multicolumn{2}{|l|}{ VLP+VLP(L) } & \multicolumn{2}{|c|}{ DNA+VLP\$ } & \multicolumn{2}{|c|}{ DNA+VLP(L3) } \\
\cline { 4 - 10 } & & & env & Gag & Env & Gag & Env & Gag & Env & gag \\
\hline Vaginal IgA & $5^{* *}$ & $5^{* *}$ & 1.7 & 1.5 & 3.56 & $\mathbf{4 0 . 3}$ & 7 & 1.5 & 8.47 & $\mathbf{7 1 . 9}$ \\
\hline Intestinal IgA & $5^{* *}$ & $5^{* *}$ & 6.05 & 8.5 & 6.5 & 6.3 & $\mathbf{1 0 . 5}$ & $\mathbf{1 2 . 2}$ & $\mathbf{1 2 . 1 5}$ & $\mathbf{1 5 . 6}$ \\
\hline
\end{tabular}

*Intra-nasal administration of 4 doses without adjuvant [16];

${ }^{* *}$ GMT obtained by ELISA performed using whole VLPS as target antigen;

$\S$ Single VLP boosting dose of 2ug. GMT obtained by ELISA performed using whole individual env or gag proteins as target antigen. 